\title{
WEAK PERTURBATIONS OF THE P-LAPLACIAN
}

\author{
TOMAS EKHOLM, RUPERT L. FRANK, AND HYNEK KOVAŘÍK
}

\begin{abstract}
We consider the p-Laplacian in $\mathbb{R}^{d}$ perturbed by a weakly coupled potential. We calculate the asymptotic expansions of the lowest eigenvalue of such an operator in the weak coupling limit separately for $p>d$ and $p=d$ and discuss the connection with Sobolev interpolation inequalities.
\end{abstract}

AMS Mathematics Subject Classification: 49R05, 35P30

Keywords: p-Laplacian, weak coupling, Sobolev inequalities

\section{INTRODUCTION}

In this paper we consider the functional

$$
Q_{V}[u]=\int_{\mathbb{R}^{d}}\left(|\nabla u|^{p}-V|u|^{p}\right) d x, \quad u \in W^{1, p}\left(\mathbb{R}^{d}\right), \quad p>1,
$$

with a given function $V: \mathbb{R}^{d} \rightarrow \mathbb{R}$ which is assumed to vanish at infinity in a sense to be made precise. We are interested in the minimization problem

$$
\lambda(V)=\inf _{u \in W^{1, p}\left(\mathbb{R}^{d}\right)} \frac{Q_{V}[u]}{\int_{\mathbb{R}^{d}}|u|^{p} d x} .
$$

If (1.2) admits a minimizer $u$, then the latter satisfies in the weak sense the non-linear eigenvalue equation

$$
-\Delta_{p}(u)-V|u|^{p-2} u=\lambda(V)|u|^{p-2} u,
$$

where $-\Delta_{p}(u):=-\nabla \cdot\left(|\nabla u|^{p-2} \nabla u\right)$ is the $p$-Laplacian. Equation (1.3) is a particular case of a quasilinear differential problem and we refer to the monographs [LU, $[\mathrm{PS}]$ and to $[\mathrm{S} 1, \mathrm{S2}, \mathrm{Tr}$ ) for the general theory of such equations. The $p$-Laplacian equation with a zero-th order term $V$ has attracted particular attention. Existence of positive solutions to the equation $-\Delta_{p}(u)=V|u|^{p-2} u$ and related regularity questions were studied in $[\mathrm{PoSh}$, PT2, TT, To, PT1. For the discussion of maximum and comparison principles and positive Liouville theorems, see [GS, PTT.

In the present paper we are going to study the behaviour of $\lambda(\alpha V)$ for small values of $\alpha$. It is not difficult to see that $\lambda(\alpha V) \rightarrow 0$ as $\alpha \rightarrow 0$ for all sufficiently regular and decaying $V$. Our goal here is to find the correct asymptotic order and the correct asymptotic coefficient.

Key words and phrases. p-Laplacian, weak coupling, Sobolev inequality.

(c) 2013 by the authors. This paper may be reproduced, in its entirety, for non-commercial purposes. 
It turns out that the asymptotic order depends essentially on the relation between the values of the exponent $p$ and the dimension $d$. If $p<d$, then by the Hardy inequality [OK] we have

$$
\int_{\mathbb{R}^{d}}|\nabla u|^{p} d x \geq\left(\frac{d-p}{p}\right)^{p} \int_{\mathbb{R}^{d}} \frac{|u|^{p}}{|x|^{p}} d x, \quad u \in W^{1, p}\left(\mathbb{R}^{d}\right), \quad d>p .
$$

Therefore, if $|V(x)| \leq C|x|^{-p}$ for some $C>0$, then $\lambda(\alpha V)=0$ for all $\alpha$ small enough. However, if $p \geq d$ and $\int_{\mathbb{R}^{d}} V>0$, then we have $\lambda(\alpha V)<0$ for any $\alpha>0$. The latter is easily verified by a suitable choice of test functions. Moreover, if $V$ is bounded and compactly supported, then $\lambda(\alpha V)<0$ for any $\alpha>0$ even when $\int_{\mathbb{R}^{d}} V=0$, see [PT1, Prop. 4.5]. Consequently, we will always assume that $p \geq p$.

The question about the asymptotic behavior of $\lambda(\alpha V)$ for small $\alpha$ was intensively studied in the linear case $p=2$ (see, e.g., BGS, Kl1, 조, Si ), where equation (1.3) defines the ground state energy of the Schrödinger operator $-\Delta-V$. In particular, it turns out that for sufficiently fast decaying $V$ we have

$$
\sqrt{-\lambda(\alpha V)}=\frac{1}{2} \alpha \int_{\mathbb{R}} V d x-c \alpha^{2}+o\left(\alpha^{2}\right), \quad \alpha \rightarrow 0, \quad d=1, p=2,
$$

with an explicit constant $c$ depending on $V$, see [Si]. The proof of (1.4) is based on the Birman-Schwinger principle and on the explicit knowledge of the unperturbed Green function. With suitable modifications, this method was applied also to Schrödinger operators with long-range potentials, BGS, K12, and even to higher order and fractional Schrödinger operators AZ1, AZ2, Ha].

Much less is known about the non-linear case $p \neq 2$ where the operator-theoretic methods developed for $p=2$ cannot be used. We will therefore apply a different, purely variational technique which allows us to analyze the asymptotic behaviour of $\lambda(\alpha V)$ for all $p>1$. A similar variational approach has already been used in a linear problem in [FMV], but here we take it much further into the quasi-linear realm (where, for instance the symmetry reduction that we crucial in [FMV] is no longer available).

We will present our main results separately for $p>d$, see Theorem 2.1] and for $p=d$, see Theorem 2.2. In the case $p>d$ we shall show, in particular, that there is a close relation between the asymptotic behaviour of $\lambda(\alpha V)$ and the Sobolev interpolation inequality (see, e.g., [Ad, Thm 5.9])

$$
\|u\|_{\infty}^{p} \leq \mathcal{S}_{d, p}\|\nabla u\|_{p}^{d}\|u\|_{p}^{p-d}, \quad u \in W^{1, p}\left(\mathbb{R}^{d}\right), \quad d<p .
$$

By convention $\mathcal{S}_{d, p}$ will always denote the optimal (that is, smallest possible) constant in (1.5). On one hand, the constant $\mathcal{S}_{d, p}$ enters into the asymptotic coefficient in the expansion of $\lambda(\alpha V)$, see equation (2.1). On the other hand, minimizers of problem (1.2), when suitably rescaled and normalised, converge (up to a subsequence) locally uniformly to a minimizer of the Sobolev inequality (1.5) as $\alpha \rightarrow 0$, see Proposition 3.7

The case $p=d$ is much more delicate and requires (slightly) more regularity of the potential $V$ since functions in $W^{1, d}\left(\mathbb{R}^{d}\right)$, which appear in (1.2), are not necessarily bounded. While the case $p>d$ can be dealt with by energy methods (i.e. on the $W^{1, p}\left(\mathbb{R}^{d}\right)$ level of regularity), heavier PDE technics (Harnack's inequality, Hölder continuity bounds) are necessary to deal with $p=d$. The subtly of the case $p=d$ can also be seen in the asymptotic 
order: while $\lambda(\alpha V)$ vanishes algebraically as $\alpha \rightarrow 0$ for $p>d$, it vanishes exponentially fast for $p=d$, see equation (2.2).

As we shall see, the asymptotic coefficient will depend on $V$ only through $\int_{\mathbb{R}^{d}} V d x$. We emphasize here that we do not impose a sign condition on $V$. Thus, the positive and the negative parts of $V$ contribute both to the asymptotic coefficient and there will be cancellations. This is one of main difficulties that we overcome. In fact, if $V$ is non-negative, then the proof is considerably simpler.

A common feature of both Theorems 2.1 and Theorem 2.2 is that their proofs rely, among other things, on the fact that minimizers $u_{\alpha}$ of (1.2), suitably normalized, converge locally uniformly to a constant. While in the case $d<p$ this follows from Morrey's Sobolev inequality and energy considerations, for $d=p$ we have to employ a regularity argument related to the Hölder continuity of $u_{\alpha}$, see Lemma 4.6, with explicit dependence on the coefficients of the equation.

\section{MAin RESUlts}

Our main results describe the asymptotics of the infimum $\lambda(\alpha V)$ of the functional $Q_{\alpha V}[u]$ as $\alpha \rightarrow 0$, see (1.1) and (1.2). Our first theorem concerns the subcritical case $p>d$.

Theorem 2.1. Let $p>d \geq 1$. Let $V \in L^{1}\left(\mathbb{R}^{d}\right)$ be such that $\int_{\mathbb{R}^{d}} V(x) d x>0$. Then

$$
\lim _{\alpha \rightarrow 0+} \alpha^{-\frac{p}{p-d}} \lambda(\alpha V)=-\frac{p-d}{p}\left(\frac{d}{p}\right)^{\frac{d}{p-d}}\left(\mathcal{S}_{d, p} \int_{\mathbb{R}^{d}} V(x) d x\right)^{\frac{p}{p-d}},
$$

where $\mathcal{S}_{d, p}$ is the sharp constant in the Sobolev inequality (1.5).

We also have a theorem that describes the asymptotics of the minimizers of the functional $Q_{\alpha V}[u]$; see Proposition 3.7 .

In the endpoint case $d=p$ we have

Theorem 2.2. Let $p=d>1$. Suppose that $V \in L^{q}\left(\mathbb{R}^{d}\right) \cap L^{1}\left(\mathbb{R}^{d}\right)$ for some $q>1$ and that $\int_{\mathbb{R}^{d}} V(x) d x>0$. Then

$$
\lim _{\alpha \rightarrow 0+} \alpha^{\frac{1}{d-1}} \log \frac{1}{|\lambda(\alpha V)|}=d \omega_{d}^{\frac{1}{d-1}}\left(\int_{\mathbb{R}^{d}} V(x) d x\right)^{-\frac{1}{d-1}},
$$

where $\omega_{d}$ denotes the surface area of the unit sphere in $\mathbb{R}^{d}$.

Remark 2.3. Let us compare the assumptions on $V$ in Theorems 2.1 and 2.2. If $p>d$ and $V_{+} \notin L^{1}\left(\mathbb{R}^{d}\right), V_{-} \in L^{1}\left(\mathbb{R}^{d}\right)$, then Theorem 2.1 easily implies that

$$
\lim _{\alpha \rightarrow 0+} \alpha^{-\frac{p}{p-d}} \lambda(\alpha V)=-\infty \text {. }
$$

Thus, at least under the additional hypothesis $V_{-} \in L^{1}\left(\mathbb{R}^{d}\right)$, the condition $V_{+} \in L^{1}\left(\mathbb{R}^{d}\right)$ is necessary and sufficient for finite asymptotics of $\alpha^{-\frac{p}{p-d}} \lambda(\alpha V)$. This is not true for the asymptotics of $\alpha^{\frac{1}{d-1}} \log |\lambda(\alpha V)|^{-1}$ in the case $p=d$, and this is the reason for the additional assumption $V \in L^{q}\left(\mathbb{R}^{d}\right)$ for some $q>1$. Indeed, we claim that there are $0 \leq V \in L^{1}\left(\mathbb{R}^{d}\right)$ such that $\lambda(\alpha V)=-\infty$ for any $\alpha>0$. To see this, choose $\sigma \in(1, d)$ and consider $V(x)=$ $|x|^{-d}|\log | x||^{-\sigma}$ for $|x| \leq e^{-1}$ and $V(x)=0$ for $|x|>e^{-1}$. Then $\sigma>1$ implies $V \in L^{1}\left(\mathbb{R}^{d}\right)$. Since $\sigma<d$ we can choose a $\rho \in[(\sigma-1) / d,(d-1) / d)$ and define $u(x)=\left.|\ln | x\right|^{\rho} \zeta(x)$, where 
the function $\zeta \in C_{0}^{\infty}\left(\mathbb{R}^{d}\right)$ equals one in a neighborhood of the origin. Then $\rho<(d-1) / d$ implies that $u \in W^{1, d}\left(\mathbb{R}^{d}\right)$, whereas $\rho \geq(\sigma-1) / d$ implies that $\int_{\mathbb{R}^{d}} V|u|^{d} d x=\infty$. Thus, $Q_{\alpha V}[u]=-\infty$ for any $\alpha>0$.

Remark 2.4. In the quadratic case $p=2$, Theorems 2.1 and 2.2 recover the asymptotics originally found in Si] using a different, operator theoretic approach. Both (2.1) and (2.2) were originally proved in [Si] under more restictive conditions on $V$. For $d=1$ these restrictions were later removed in [K11, Sec.4]; note also that according to Lemma 3.3 below we have $\mathcal{S}_{1,2}=1$ for $p=2$ and $d=1$.

While our theorems give a complete answer in the case $V \in L^{1}\left(\mathbb{R}^{d}\right)$ (plus additional assumptions if $p=d$ ) with $\int_{\mathbb{R}^{d}} V d x>0$, the following questions, which we consider interesting, remain open:

(1) What happens if $V \in L^{1}\left(\mathbb{R}^{d}\right)$ (plus some additional assumptions), but $\int_{\mathbb{R}^{d}} V d x=0$ ? For results in the case $p=2$, see [Si, Kl1, BCEZ].

(2) What happens if $V \notin L^{1}\left(\mathbb{R}^{d}\right)$, but $V(x)=|x|^{-\sigma}(1+o(1))$ as $|x| \rightarrow \infty$ with $0<\sigma \leq d$ ? For results in the case $p=2$, see $[\mathrm{K} 12$.

The proofs of Theorems 2.1 and 2.2 are given in Sections 3 and 4 respectively.

Notation. Given $r>0$ and a point $x \in \mathbb{R}^{d}$ we denote by $B(r, x) \subset \mathbb{R}^{d}$ the open ball with radius $r$ centred in $x$. If $x=0$, then we write $B_{r}$ instead of $B(r, 0)$. Furthermore, given a set $\Omega \subset \mathbb{R}^{d}$ we denote by $\Omega^{c}$ its complement in $\mathbb{R}^{d}$. The $L^{q}$ norm of a function $u$ in $\Omega$ will be denoted by $\|u\|_{L^{q}(\Omega)}$ if $\Omega \neq \mathbb{R}^{d}$ and by $\|u\|_{q}$ if $\Omega=\mathbb{R}^{d}$.

\section{CASE $d<p$}

Before we proceed with the proof of Theorem 2.1 we give some preliminary results concerning Sobolev inequality (1.5) and the properties of the functional $Q_{V}[u]$.

3.1. Sobolev inequality. We recall that $\mathcal{S}_{d, p}$ denotes the optimal constant in the Sobolev interpolation inequality (1.5). In this subsection we discuss a closely related (and, in fact, equivalent, as we shall show) minimization problem which depends on a parameter $v>0$ in addition to an exponent $q>d \geq 1$. We define

$$
E(v)=\inf _{\|u\|_{p}=1}\left(\|\nabla u\|_{p}^{p}-v|u(0)|^{p}\right) .
$$

(Note that by the Sobolev embedding theorem any function in $W^{1, q}\left(\mathbb{R}^{d}\right), q>d$, has a continuous representative and therefore $u(0)$ is unambiguously defined. The following lemma shows, in particular, that $E(v)>-\infty$.

Lemma 3.1. Let $p>d \geq 1$ and $v>0$. Then

$$
E(v)=-\frac{p-d}{p}\left(\frac{d}{p}\right)^{\frac{d}{p-d}}\left(\mathcal{S}_{d, p} v\right)^{\frac{p}{p-d}} .
$$

Moreover, the infimum is attained by a non-negative, symmetric decreasing function. Finally, any minimizing sequence is relatively compact in $W^{1, p}\left(\mathbb{R}^{d}\right)$.

We include a proof of this lemma for the sake of completeness. 
Proof. By the Sobolev inequality (1.5) we have

$$
|u(0)|^{p} \leq\|u\|_{\infty}^{p} \leq \mathcal{S}_{d, p}\|\nabla u\|_{p}^{d}\|u\|_{p}^{p-d}
$$

and, therefore, if $\|u\|_{p}=1$,

$$
\begin{aligned}
\|\nabla u\|_{p}^{p}-v|u(0)|^{p} \geq\|\nabla u\|_{p}^{p}-v \mathcal{S}_{d, p}\|\nabla u\|_{p}^{d} & \geq \inf _{X \geq 0}\left(X^{p}-v \mathcal{S}_{d, p} X^{d}\right) \\
& =-\frac{p-d}{p}\left(\frac{d}{p}\right)^{\frac{d}{p-d}}\left(\mathcal{S}_{d, p} v\right)^{\frac{p}{p-d}} .
\end{aligned}
$$

This shows that $E(v) \geq-\frac{p-d}{p}\left(\frac{d}{p}\right)^{\frac{d}{p-d}}\left(\mathcal{S}_{d, p} v\right)^{\frac{p}{p-d}}$. In particular, $E(v)>-\infty$.

To prove the reverse inequality, we first note that, by scaling,

$$
E(v)=E(1) v^{\frac{p}{p-d}} .
$$

(To see this, write $u$ in the form $u(x)=v^{\frac{d}{p(p-d)}} w\left(v^{\frac{1}{p-d}} x\right)$.) We note also that $E(v)<0$. (Indeed, for a fixed $u \in W^{1, p}\left(\mathbb{R}^{d}\right)$ with $\|u\|_{p}=1$ and $u(0) \neq 0$ we clearly have $\|\nabla u\|_{p}^{p}-$ $v|u(0)|^{p} \rightarrow-\infty$ as $v \rightarrow \infty$ and therefore $E(v)<0$ for all sufficiently large $v$. By the scaling law, this implies that $E(v)<0$ for any $v$.)

Now let $u \in W^{1, p}\left(\mathbb{R}^{d}\right)$. Then, by the Sobolev embedding theorem $u$ can be assumed to be continuous and vanishing at infinity, so there is an $a \in \mathbb{R}^{d}$ such that $|u(a)|=\|u\|_{\infty}$. Let $\tilde{u}(x)=u(x+a) /\|u\|_{p}$. Then, by the definition of $E(v)$,

$$
\|\nabla \tilde{u}\|_{p}^{p}-v|\tilde{u}(0)|^{p} \geq E(v),
$$

i.e.,

$$
\|\nabla u\|_{p}^{p} \geq v\|u\|_{\infty}^{p}+E(v)\|u\|_{p}^{p}=v\|u\|_{\infty}^{p}+E(1) v^{\frac{p}{p-d}}\|u\|_{p}^{p} .
$$

Since this is true for any $v>0$ we have

$$
\begin{aligned}
\|\nabla u\|_{p}^{p} \geq v\|u\|_{\infty}^{p}+E(v)\|u\|_{p}^{p} & \geq \sup _{v>0}\left(v\|u\|_{\infty}^{p}+E(1) v^{\frac{p}{p-d}}\|u\|_{p}^{p}\right) \\
& =\|u\|_{\infty}^{\frac{p^{2}}{d}}\|u\|_{p}^{-\frac{p(p-d)}{d}}|E(1)|^{-\frac{p-d}{d}}\left(\frac{p-d}{p}\right)^{\frac{p-d}{d}} \frac{d}{p}
\end{aligned}
$$

This proves that $\mathcal{S}_{d, p} \leq|E(1)|^{\frac{p-d}{p}}\left(\frac{p-d}{p}\right)^{-\frac{p-d}{p}}\left(\frac{d}{p}\right)^{\frac{d}{p}}$.

We next prove that any minimizing sequence is relatively compact in $W^{1, p}\left(\mathbb{R}^{d}\right)$. Let $\left(u_{n}\right) \subset W^{1, p}\left(\mathbb{R}^{d}\right)$ be a minimizing sequence for $E(v)$. Using the bounds in the first part of the proof it is easy to see that $\left(u_{n}\right)$ is bounded in $W^{1, p}\left(\mathbb{R}^{d}\right)$ and therefore, after passing to a subsequence if necessary, we may assume that $u_{n}$ converges weakly in $W^{1, p}\left(\mathbb{R}^{d}\right)$ to some $u \in W^{1, p}\left(\mathbb{R}^{d}\right)$. By weak convergence,

$$
\liminf _{n \rightarrow \infty}\left\|\nabla u_{n}\right\|_{p}^{p} \geq\|\nabla u\|_{p}^{p}, \quad 1 \geq \liminf _{n \rightarrow \infty}\left\|u_{n}\right\|_{p}^{p} \geq\|u\|_{p}^{p}
$$

and, by the Rellich-Kondrashov theorem (see, e.g., [LL, Thm. 8.9]), $u_{n}(0) \rightarrow u(0)$. We conclude that

$$
0>E(v)=\lim _{n \rightarrow \infty}\left(\left\|\nabla u_{n}\right\|_{p}^{p}-v\left|u_{n}(0)\right|^{p}\right) \geq\|\nabla u\|_{p}^{p}-v|u(0)|^{p} \geq E(v)\|u\|_{p}^{p} .
$$


This, together with the second assertion in (3.2) implies that $\|u\|_{p}=1$. Together with the first assertion in (3.2) and the convergence of $u_{n}(0)$ it also implies that $\left\|\nabla u_{n}\right\|_{p} \rightarrow\|\nabla u\|_{p}$. Thus, $u_{n}$ converges in fact strongly to $u$ in $W^{1, p}\left(\mathbb{R}^{d}\right)$.

Thus, we have shown that there is a minimizer. In view of the rearrangement inequalities $\left\|\nabla u^{*}\right\|_{p} \leq\|\nabla u\|_{p},\left\|u^{*}\right\|_{p}=\|u\|_{p}$ and $\left|u^{*}(0)\right| \geq|u(0)|$ (see, e.g., [Ta] and [LL, Thm. 3.4]) we see that among the minimizers there is a non-negative, symmetric decreasing function. This concludes the proof.

Remark 3.2. It is easy to see that

$$
E(v)=\inf _{\|u\|_{p}=1}\left(\|\nabla u\|_{p}^{p}-v\|u\|_{\infty}^{p}\right) .
$$

This will be useful in the following.

In one dimension we can compute the value of the sharp constant $\mathcal{S}_{d, p}$ in (1.5).

Lemma 3.3. If $d=1$, then $\mathcal{S}_{1, p}=\frac{p}{2}$ for any $p>1$.

Proof. Let $u$ be the (symmetric decreasing) optimizer for $E(v)$. The Euler-Lagrange equation reads

$$
(p-1) u^{\prime \prime}(x)\left(-u^{\prime}(x)\right)^{p-2}=\lambda u(x)^{p-1} \quad \text { in }(0, \infty),
$$

together with the boundary condition

$$
2\left(-u^{\prime}(0+)\right)^{p-1}=v u(0)^{p-1} .
$$

Multiplying (3.3) by $u^{\prime}$ we obtain

$$
\left((p-1)\left(-u^{\prime}\right)^{p}-\lambda u^{p}\right)^{\prime}=0 \quad \text { in }(0, \infty) .
$$

Since $u \in W^{1, p}\left(\mathbb{R}^{d}\right)$ we have $u(x) \rightarrow 0$ as $x \rightarrow \infty$. Since $(p-1)\left(-u^{\prime}\right)^{p}-\lambda u^{p}$ is constant, $\lim _{x \rightarrow \infty} u^{\prime}(x)$ exists as well and, therefore, needs to be zero. Thus

$$
(p-1)\left(-u^{\prime}\right)^{p}-\lambda u^{p}=0 \quad \text { in }(0, \infty) .
$$

Note that this shows that $\lambda>0$. Moreover, we obtain

$$
-u^{\prime}=\left(\frac{\lambda}{p-1}\right)^{\frac{1}{p}} u \quad \text { in }(0, \infty),
$$

and, thus,

$$
u(x)=u(0) \exp \left(-\left(\frac{\lambda}{p-1}\right)^{\frac{1}{p}} x\right) \quad \text { in }(0, \infty) .
$$

The boundary condition implies that $\lambda=(p-1)(v / 2)^{p /(p-1)}$. We conclude that

$$
E(v)=\frac{2 \int_{0}^{\infty}\left|u^{\prime}\right|^{p} d x-v u(0)^{p}}{2 \int_{0}^{\infty} u^{p} d x}=-(p-1)\left(\frac{v}{2}\right)^{\frac{p}{p-1}} .
$$

By Lemma 3.1 this implies the assertion. 


\subsection{Preliminaries.}

Lemma 3.4. Let $p>d$ and assume that $V \in L^{1}\left(\mathbb{R}^{d}\right)$. Then for any $u \in W^{1, p}\left(\mathbb{R}^{d}\right)$,

$$
Q_{V}[u] \geq-\frac{p-d}{p}\left(\frac{d}{p}\right)^{\frac{d}{p-d}}\left(\mathcal{S}_{d, p} \int_{\mathbb{R}^{d}} V_{+} d x\right)^{\frac{p}{p-d}}\|u\|_{p}^{p} .
$$

Moreover, $Q_{V}[u]$ is weakly lower semi-continuous in $W^{1, p}\left(\mathbb{R}^{d}\right)$.

Proof. For any $u \in W^{1, p}\left(\mathbb{R}^{d}\right)$,

$$
Q_{V}[u] \geq\|\nabla u\|_{p}^{p}-\int_{\mathbb{R}^{d}} V_{+} d x\|u\|_{\infty}^{p} \geq E\left(\int_{\mathbb{R}^{d}} V_{+} d x\right) .
$$

The second inequality used Remark 3.2. The first assertion now follows from Lemma 3.1 .

To prove weak lower semi-continuity assume that $\left(u_{j}\right)$ converges weakly in $W^{1, p}\left(\mathbb{R}^{d}\right)$ to some $u$. Then the sequence $\left(u_{j}\right)$ is bounded in $W^{1, p}\left(\mathbb{R}^{d}\right)$ and hence, by (1.5) $)$ in $L^{\infty}\left(\mathbb{R}^{d}\right)$. We have

$$
\left|\int_{\mathbb{R}^{d}} V\left(\left|u_{j}\right|^{p}-|u|^{p}\right) d x\right| \leq\left\|u_{j}-u\right\|_{L^{\infty}\left(B_{R}\right)}\left\|f_{j}\right\|_{\infty}\|V\|_{1}+2\left(\sup _{j}\left\|u_{j}\right\|_{\infty}^{p}\right)\|V\|_{L^{1}\left(B_{R}^{c}\right)},
$$

where $f_{j}:=\left(\left|u_{j}\right|^{p}-|u|^{p}\right) /\left(\left|u_{j}\right|-|u|\right)$ satisfies $\left|f_{j}\right| \leq p \max \left\{\left|u_{j}\right|^{p-1},|u|^{p-1}\right\}$ and is therefore bounded. Since the sequence $\left(u_{j}\right)$ is bounded in $W^{1, p}\left(\mathbb{R}^{d}\right)$, inequality (1.5) implies that $\left\|f_{j}\right\|_{\infty}$ is bounded uniformly with respect to $j$. On the other hand, the Rellich-Kondrashov theorem (see, e.g., [LL, Thm.8.9]) says that $\left(u_{j}\right)$ converges to $u$ uniformly on compact subsets of $\mathbb{R}^{d}$. Hence, sending first $j \rightarrow \infty$ and then $R \rightarrow \infty$ in (3.6) shows that the functional $\int_{\mathbb{R}^{d}} V|u|^{p} d x$ is weakly continuous on $W^{1, p}\left(\mathbb{R}^{d}\right)$. Since $\|\nabla u\|_{p}^{p}$ is weakly lower semi-continuous, due to the fact that $p>1$, the same is true for $Q_{V}[u]$.

Remark 3.5. Note that inequality (3.5) yields the lower bound in (2.1) in the case $V \geq 0$.

Corollary 3.6. Let $V \in L^{1}\left(\mathbb{R}^{d}\right)$ and $p>d$. Assume that $\lambda(V)<0$. Then there is a non-negative function $u \in W^{1, p}\left(\mathbb{R}^{d}\right)$ such that

$$
\lambda(V)=\frac{Q_{V}[u]}{\|u\|_{p}^{p}} .
$$

Proof. Let $\left(u_{j}\right)$ be a minimizing sequence for $Q_{V}$, normalized such that $\left\|u_{j}\right\|_{p}=1$ for any $j \in \mathbb{N}$. Since $\lambda(V)<0$, we may assume without loss of generality that $Q_{V}\left[u_{j}\right]<0$ for any $j \in \mathbb{N}$. Hence with the help of (1.5) we get

$$
\left\|\nabla u_{j}\right\|_{p}^{p}<\int_{\mathbb{R}^{d}} V_{+}\left|u_{j}\right|^{p} d x \leq\left\|V_{+}\right\|_{1}\left\|u_{j}\right\|_{\infty}^{p} \leq \mathcal{S}_{d, p}\left\|V_{+}\right\|_{1}\left\|\nabla u_{j}\right\|_{p}^{d} .
$$

Since $p>d$, it follows that the sequence $\left(u_{j}\right)$ is bounded in $W^{1, p}\left(\mathbb{R}^{d}\right)$ and, after passing to a subsequence if necessary, we may assume that $\left(u_{j}\right)$ converges weakly in $W^{1, p}\left(\mathbb{R}^{d}\right)$ to some $u \in W^{1, p}\left(\mathbb{R}^{d}\right)$. The weak convergence implies

$$
\|u\|_{p} \leq \liminf _{j \rightarrow \infty}\left\|u_{j}\right\|_{p}=1
$$

Since $Q_{V}[u]$ is weakly lower semicontinuous by Lemma 3.4, the above inequality implies

$$
0>\lambda(V)=\lim _{j \rightarrow \infty} Q_{V}\left[u_{j}\right] \geq Q_{V}[u] \geq \lambda(V)\|u\|_{p}^{p} \geq \lambda(V) .
$$


This implies that $Q_{V}[u]=\lambda(V)$ and $\|u\|_{p}=1$, i.e., $u$ is a minimizer for the problem (1.2).

Since $u \in W^{1, p}\left(\mathbb{R}^{d}\right)$ implies $|u| \in W^{1, p}\left(\mathbb{R}^{d}\right)$ with $|\nabla| u||=|\nabla u|$ almost everywhere (see, e.g., [LL, Thm. 6.17]), we may choose $u$ non-negative.

3.3. Proof of Theorem 2.1. Upper bound. For any fixed function $\varphi \in W^{1, p}\left(\mathbb{R}^{d}\right)$ with $\|\varphi\|_{p}=1$ we define

$$
v_{\alpha}(x):=\alpha^{\frac{d}{p(p-d)}} \varphi\left(\alpha^{\frac{1}{p-d}} x\right), \quad \alpha>0 .
$$

Then $\left\|v_{\alpha}\right\|_{p}=1$ for all $\alpha>0$ and

$$
\lambda(\alpha V) \leq Q_{\alpha V}\left[v_{\alpha}\right]=\alpha^{\frac{p}{p-d}}\left(\|\nabla \varphi\|_{p}^{p}-\int_{\mathbb{R}^{d}} V(x)\left|\varphi\left(\alpha^{\frac{1}{p-d}} x\right)\right|^{p} d x\right) .
$$

Since $\varphi \in W^{1, p}\left(\mathbb{R}^{d}\right)$, the Sobolev embedding implies that $\varphi \in C\left(\mathbb{R}^{d}\right) \cap L^{\infty}\left(\mathbb{R}^{d}\right)$ and therefore, by dominated convergence,

$$
\int_{\mathbb{R}^{d}} V(x)\left|\varphi\left(\alpha^{\frac{1}{p-d}} x\right)\right|^{p} d x \rightarrow \int_{\mathbb{R}^{d}} V d x|\varphi(0)|^{p} \quad \text { as } \alpha \rightarrow 0 .
$$

Since $\varphi$ is arbitrary, we have shown that

$$
\limsup _{\alpha \rightarrow 0+} \alpha^{\frac{p}{d-p}} \lambda(\alpha V)=\inf _{\|\varphi\|_{p}=1}\left(\|\nabla \varphi\|_{p}^{p}-\int_{\mathbb{R}^{d}} V d x|\varphi(0)|^{p}\right)=E\left(\int_{\mathbb{R}^{d}} V d x\right) .
$$

The upper bound in Theorem 2.1 now follows from Lemma 3.1

3.4. Proof of Theorem 2.1. Lower bound. It follows from the proof of the upper bound that $\lambda(\alpha V)<0$ for all sufficiently small $\alpha>0$ and hence, by Corollary 3.6, for all such $\alpha$ there is a non-negative minimizer $u_{\alpha}$ of the problem (1.2). (It is easy to see that, in fact, $\lambda(\alpha V)<0$ for all $\alpha>0$. Indeed, $\alpha^{-1} Q_{\alpha V}[u]$ is non-increasing for every $u \in W^{1, p}\left(\mathbb{R}^{d}\right)$ and therefore $\alpha^{-1} \lambda(\alpha V)$ is non-increasing. Thus, if it is negative for some $\alpha>0$, it is negative for all larger $\alpha$ 's.)

We normalize $u_{\alpha}$ so that $\left\|u_{\alpha}\right\|_{p}=1$. The key step in the proof is to show that

$$
\lim _{\alpha \rightarrow 0+} \alpha^{-\frac{d}{p-d}} \int_{\mathbb{R}^{d}} V(x)\left(u_{\alpha}(x)^{p}-u_{\alpha}(0)^{p}\right) d x=0 .
$$

Assuming this for the moment, let us complete the proof. We define

$$
f_{\alpha}(x)=\alpha^{-\frac{d}{p(p-d)}} u_{\alpha}\left(x \alpha^{-\frac{1}{p-d}}\right)
$$

and observe that $\left\|f_{\alpha}\right\|_{p}=1$ and

$$
\left\|\nabla f_{\alpha}\right\|_{p}^{p}-\int_{\mathbb{R}^{d}} V_{\alpha}(x) f_{\alpha}(x)^{p} d x=\alpha^{-\frac{p}{p-d}} Q_{\alpha V}\left[u_{\alpha}\right],
$$

where $V_{\alpha}(x)=\alpha^{-d /(p-d)} V\left(x \alpha^{-1 /(p-d)}\right)$. Since (3.9) can be rewritten as

$$
\lim _{\alpha \rightarrow 0}\left(\int_{\mathbb{R}^{d}} V_{\alpha}(x) f_{\alpha}(x)^{p} d x-\int_{\mathbb{R}^{d}} V d x f_{\alpha}(0)^{p}\right)=0,
$$


we obtain

$$
\begin{aligned}
\liminf _{\alpha \rightarrow 0+} \alpha^{-\frac{p}{p-d}} \lambda(\alpha V) & =\liminf _{\alpha \rightarrow 0+} \alpha^{-\frac{p}{p-d}} Q_{\alpha V}\left[u_{\alpha}\right] \\
& =\liminf _{\alpha \rightarrow 0+}\left(\left\|\nabla f_{\alpha}\right\|_{p}^{p}-\int_{\mathbb{R}^{d}} V d x f_{\alpha}(0)^{p}\right) \\
& \geq E\left(\int_{\mathbb{R}^{d}} V d x\right) \\
& =-\frac{p-d}{p}\left(\frac{d}{p}\right)^{\frac{d}{p-d}}\left(\mathcal{S}_{d, p} \int_{\mathbb{R}^{d}} V(x) d x\right)^{\frac{p}{p-d}} .
\end{aligned}
$$

The last equality comes from Lemma 3.1. This is the lower bound claimed in Theorem 2.1,

It remains to prove (3.9). Arguing as in (3.8) we obtain $\left\|\nabla u_{\alpha}\right\|_{p}^{p} \leq \alpha \mathcal{S}_{d, p}\left\|V_{+}\right\|\left\|_{1}\right\| \nabla u_{\alpha} \|_{p}^{d}$, and therefore

$$
\left\|\nabla u_{\alpha}\right\|_{p} \leq C \alpha^{\frac{1}{p-d}}
$$

According to (1.5) this also implies

$$
\left\|u_{\alpha}\right\|_{\infty}^{p} \leq C^{\prime} \alpha^{\frac{d}{p-d}} .
$$

By Morrey's Sobolev inequality there is a constant $\mathcal{M}=\mathcal{M}_{d, p}$ such that for all $v \in W^{1, p}\left(\mathbb{R}^{d}\right)$ and all $x, y \in \mathbb{R}^{d}$ one has

$$
|v(x)-v(y)| \leq \mathcal{M}|x-y|^{(p-d) / p}\|\nabla v\|_{p} .
$$

We now fix $R>0$ and use Morrey's inequality (3.14) together with (3.12) to get for all $x \in B_{R}$

$$
\left|u_{\alpha}(x)-u_{\alpha}(0)\right| \leq \mathcal{M} R^{\frac{p-d}{p}}\left\|\nabla u_{\alpha}\right\|_{p} \leq C_{R} \alpha^{\frac{1}{p-d}}
$$

This, together with (3.13), yields for all $x \in B_{R}$

$$
\left|u_{\alpha}(x)^{p}-u_{\alpha}(0)^{p}\right| \leq p\left|u_{\alpha}(x)-u_{\alpha}(0)\right| \max \left\{u_{\alpha}(x)^{p-1}, u_{\alpha}(0)^{p-1}\right\} \leq C_{R}^{\prime} \alpha^{\frac{p+d(p-1)}{p(p-d)}}
$$

Thus,

$$
\begin{aligned}
& \alpha^{-\frac{d}{p-d}}\left|\int_{\mathbb{R}^{d}} V(x)\left(u_{\alpha}(x)^{p}-u_{\alpha}(0)^{p}\right) d x\right| \\
& \quad \leq \alpha^{-\frac{d}{p-d}}\|V\|_{1} \sup _{B_{R}}\left|u_{\alpha}^{p}-u_{\alpha}(0)^{p}\right|+\alpha^{-\frac{d}{p-d}} 2\left\|u_{\alpha}\right\|_{\infty}^{p} \int_{B_{R}^{c}}|V| d x \\
& \quad \leq \alpha^{\frac{1}{p}} C_{R}^{\prime}\|V\|_{1}+2 C^{\prime} \int_{B_{R}^{c}}|V| d x .
\end{aligned}
$$

Letting first $\alpha \rightarrow 0$ and then $R \rightarrow \infty$ we obtain (3.9). This completes the proof.

3.5. Convergence of minimizers. The following theorem about the behavior of the $u_{\alpha}$ is an (almost) immediate consequence of Lemma 3.1 and Theorem 2.1 and its proof.

Proposition 3.7. Let $p>d$ and let $V \in L^{1}\left(\mathbb{R}^{d}\right)$ with $\int_{\mathbb{R}^{d}} V(x) d x>0$. For $\alpha>0$ let $u_{\alpha}$ be a non-negative minimizer of $Q_{\alpha V}[\cdot]$ with $\left\|u_{\alpha}\right\|_{p}=1$ and define $f_{\alpha}$ by (3.10). Then for any sequence $\left(\alpha_{n}\right) \subset(0, \infty)$ converging to zero there is a subsequence $\left(\alpha_{n_{k}}\right)$ and an $f_{0} \in W^{1, p}\left(\mathbb{R}^{d}\right)$ such that $f_{\alpha_{n_{k}}} \rightarrow f_{0}$ in $W^{1, p}\left(\mathbb{R}^{d}\right)$. Moreover, $f_{0}$ is a minimizer of (3.1) with $v=\int_{\mathbb{R}^{d}} V d x$. 
We recall that, by the Sobolev embedding theorem and the Rellich-Kondrachov theorem, convergence in $W^{1, p}\left(\mathbb{R}^{d}\right)$ for $p>d$ implies convergence in $L^{\infty}\left(\mathbb{R}^{d}\right)$ and in $C^{0,(p-d) / p}\left(\mathbb{R}^{d}\right)$.

We also note that if the minimizer of the Sobolev inequality (1.5) is unique (up to translations, dilations and multiplication by constants), then Proposition 3.7 implies that $f_{\alpha}$ converges as $\alpha \rightarrow 0$ (without passing to a subsequence).

Proof. It follows from (3.11) together with the upper bound in Theorem 2.1 that $\left(f_{\alpha}\right)$ is a minimizing sequence for problem (3.1) with $v=\int_{\mathbb{R}^{d}} V d x$. Therefore, the assertion follows from the relative compactness asserted in Lemma 3.1 .

\section{CASE $d=p$}

Throughout this section we suppose that $p=d$. Similarly as in the case $d<p$ we start with a couple of preliminary lemmas which which will be used to ensure existence of a minimizer of problem (1.2).

\subsection{Preliminary results.}

Lemma 4.1. Assume that $V \in L^{q}\left(\mathbb{R}^{d}\right)$ with some $q>1$. Then $Q_{V}[u] /\|u\|_{d}^{d}$ is bounded from below and $Q_{V}[\cdot]$ is weakly lower semi-continuous in $W^{1, p}\left(\mathbb{R}^{d}\right)$.

Recall that by Sobolev inequalities, see, e.g., $[\mathrm{Ad}]$, for every $r \in[d, \infty)$ there is a constant $\tilde{\mathcal{S}}_{d, r}$ such that

$$
\|u\|_{r} \leq \tilde{\mathcal{S}}_{d, r}\|\nabla u\|_{d}^{\theta}\|u\|_{d}^{1-\theta}, \quad \text { for all } u \in W^{1, d}\left(\mathbb{R}^{d}\right) .
$$

Here $0 \leq \theta<1$ is defined by $\frac{d}{r}=1-\theta$.

Proof. Hölder's inequality and (4.1) with $r=d q /(q-1)$ imply that

$$
\int_{\mathbb{R}^{d}} V|u|^{d} d x \leq\left\|V_{+}\right\|_{q}\|u\|_{r}^{d} \leq\left\|V_{+}\right\|_{q} \tilde{\mathcal{S}}_{d, r}\|\nabla u\|_{d}^{d \theta}\|u\|_{d}^{d(1-\theta)} .
$$

Thus,

$$
\begin{aligned}
Q_{V}[u] & \geq\|\nabla u\|_{d}^{d}-\left\|V_{+}\right\|_{q} \tilde{\mathcal{S}}_{d, r}\|\nabla u\|_{d}^{d \theta}\|u\|_{d}^{d(1-\theta)} \\
& \geq \inf _{X \geq 0}\left(X-\left\|V_{+}\right\|_{q} \tilde{\mathcal{S}}_{d, r} X^{\theta}\|u\|_{d}^{d(1-\theta)}\right) \\
& \geq-C\left\|V_{+}\right\|_{q}^{\frac{1}{1-\theta}}\|u\|_{d}^{d}
\end{aligned}
$$

where $C>0$ depends only on $d$ and $q$ (through $r$ ). This proves lower boundedness.

Now let us prove weak lower semi-continuity of $Q_{V}[u]$. As in the proof of Lemma 3.4 it suffices to show that $\int_{\mathbb{R}^{d}} V|u|^{p} d x$ is weakly continuous on $W^{1, d}\left(\mathbb{R}^{d}\right)$. Assume that $\left(u_{j}\right)$ converges weakly in $W^{1, d}\left(\mathbb{R}^{d}\right)$ to some $u$. Given $\delta>0$ define $\Omega_{\delta}=\left\{x \in \mathbb{R}^{d}:|V(x)|>\delta\right\}$. Since $\left(u_{j}\right)$ is bounded in $L^{d}\left(\mathbb{R}^{d}\right)$, we have

$$
\left|\int_{\Omega_{\delta}^{c}} V\left(|u|^{d}-\left|u_{j}\right|^{d}\right) d x\right| \leq C \delta
$$


with $C$ independent of $j$. Moreover, the Sobolev inequality (4.1) implies that $u_{j}$ is uniformly bounded in $L^{r}\left(\mathbb{R}^{d}\right)$ for every $r \in[d, \infty)$. Hence by Hölder inequality

$$
\begin{aligned}
\left|\int_{\Omega_{\delta}} V\left(|u|^{d}-\left|u_{j}\right|^{d}\right) d x\right| & \leq\|V\|_{q}\left(\left.\int_{\Omega_{\delta}}|| u\right|^{d}-\left|u_{j}\right|^{\left.d\right|^{\frac{q}{q-1}}} d x\right)^{\frac{q-1}{q}} \\
& =\|V\|_{q}\left(\int_{\Omega_{\delta}}\left|\left(|u|-\left|u_{j}\right|\right) \varphi_{j}\right|^{\frac{q}{q-1}} d x\right)^{\frac{q-1}{q}},
\end{aligned}
$$

where for every $r \in[d, \infty)$ there is a $C_{r}$ such that $\left\|\varphi_{j}\right\|_{r} \leq C_{r}$ for all $j$. Since $\Omega_{\delta}$ has finite measure, $u_{j} \rightarrow u$ in $L^{r}\left(\Omega_{\delta}\right)$ for any $r<\infty$ by the Rellich-Kondrashov theorem. (For instance, in [LL, Thm. 8.9], the Rellich-Kondrashov theorem is only stated for bounded sets. However, for any $\varepsilon>0$ we can find a bounded set $\omega \subset \Omega_{\delta}$ such that $\left|\Omega_{\delta} \backslash \omega\right|<\varepsilon$. Then $u_{j} \rightarrow u$ in $L^{r}(\omega)$ by the bounded Rellich-Kondrashov theorem and, since $\left(u_{j}\right)$ is bounded in $L^{s}\left(\Omega_{\delta}\right)$ for some $s>r$, by Hölder $\left\|u_{j}\right\|_{L^{r}\left(\Omega_{\delta} \backslash \omega\right)} \leq\left\|u_{j}\right\|_{L^{s}\left(\Omega_{\delta}\right)} \varepsilon^{(s-r) / s}$. Thus, $u_{j} \rightarrow u$ in $L^{r}\left(\Omega_{\delta}\right)$, as claimed.)

We thus conclude, again with $r=2 q /(q-1)$, that

$$
\int_{\Omega_{\delta}}\left|\left(|u|-\left|u_{j}\right|\right) \varphi_{j}\right|^{\frac{q}{q-1}} d x \leq C_{r}^{\frac{q}{q-1}}\left(\int_{\Omega_{\delta}}\left|u-u_{j}\right|^{\frac{2 q}{q-1}} d x\right)^{1 / 2} \rightarrow 0 \quad \text { as } j \rightarrow \infty .
$$

This in combination with (4.2) proves the claimed weak continuity.

\subsection{Proof of Theorem 2.2. Upper bound.}

Proposition 4.2. Let $V \in L^{1}\left(\mathbb{R}^{d}\right)$ be such that $\int_{\mathbb{R}^{d}} V(x) d x>0$. Then

$$
\limsup _{\alpha \rightarrow 0+} \alpha^{\frac{1}{d-1}} \log \frac{1}{|\lambda(\alpha V)|} \leq d \omega_{d}^{\frac{1}{d-1}}\left(\int_{\mathbb{R}^{d}} V(x) d x\right)^{-\frac{1}{d-1}} .
$$

Proof. Let $\beta>1$ and consider the family of test functions $v_{\beta}$ defined by

$$
v_{\beta}(x)=1 \quad \text { if } \quad|x| \leq 1, \quad v_{\beta}(x)=\left(1-\frac{\log |x|}{\log \beta}\right)_{+} \quad \text { if }|x|>1 .
$$

Then $v_{\beta} \in W^{1, d}\left(\mathbb{R}^{d}\right)$ and, since $0 \leq v_{\beta} \leq \chi_{\{|\cdot|<\beta\}}$, we have

$$
\left\|v_{\beta}\right\|_{d}^{d} \leq c \beta^{d}
$$

for all $\beta>1$ with a constant $c>0$ depending only on $d$. Moreover,

$$
Q_{\alpha V}\left[v_{\beta}\right] \leq \omega_{d}(\log \beta)^{1-d}-\alpha \int_{\mathbb{R}^{d}} V(x) d x+\alpha R_{\beta}
$$

with

$$
R_{\beta}=\int_{\{|x|>1\}} V_{+}\left(1-\left(1-\frac{\log |x|}{\log \beta}\right)_{+}\right) d x .
$$

By dominated convergence, $R_{\beta} \rightarrow 0$ as $\beta \rightarrow \infty$.

Let $\varepsilon>0$ be given and choose $\beta_{\varepsilon}>1$ such that

$$
R_{\beta} \leq \varepsilon \int_{\mathbb{R}^{d}} V d x \quad \text { for all } \beta \geq \beta_{\varepsilon} .
$$


Now, for any $\alpha$, define

$$
\beta(\alpha)=\exp \left(\left(\frac{\omega_{d}}{\alpha(1-\varepsilon) \int_{\mathbb{R}^{d}} V d x}\right)^{1 /(d-1)}\right) .
$$

Note that $\beta(\alpha)>1$ and that

$$
\frac{\omega_{d}}{(\log \beta(\alpha))^{d-1}}-\alpha(1-\varepsilon) \int_{\mathbb{R}^{d}} V d x=0 .
$$

Define $\alpha_{\varepsilon}>0$ by $\beta\left(\alpha_{\varepsilon}\right)=\beta_{\varepsilon}$. Then for $\alpha \leq \alpha_{\varepsilon}$ our upper bound on $Q_{\alpha V}\left[v_{\beta}\right]$ is non-positive and therefore

$$
\begin{aligned}
\lambda(\alpha V) & \leq \frac{Q_{\alpha V}\left[v_{\beta(\alpha)}\right]}{\left\|u_{\beta(\alpha)}\right\|_{d}^{d}} \\
& \leq c^{-1} \beta(\alpha)^{-d}\left(\omega_{d}(\log \beta(\alpha))^{1-d}-\alpha \int_{\mathbb{R}^{d}} V(x) d x+\alpha R_{\beta}\right) \\
& =-c^{-1} \alpha\left(\varepsilon \int_{\mathbb{R}^{d}} V d x-R_{\beta(\alpha)}\right) \exp \left(-d\left(\frac{\omega_{d}}{\alpha(1-\varepsilon) \int_{\mathbb{R}^{d}} V d x}\right)^{1 /(d-1)}\right) .
\end{aligned}
$$

This implies

$$
\limsup _{\alpha \rightarrow 0+} \alpha^{\frac{1}{d-1}} \log \frac{1}{|\lambda(\alpha V)|} \leq d \omega_{d}^{\frac{1}{d-1}}\left((1-\varepsilon) \int_{\mathbb{R}^{d}} V(x) d x\right)^{-\frac{1}{d-1}} .
$$

By letting $\varepsilon \rightarrow 0$ we arrive at (4.3).

Corollary 4.3. Let $V$ satisfy assumptions of Lemma 4.1. Then for every $\alpha>0$ there exists a locally bounded positive function $u_{\alpha} \in W^{1, d}\left(\mathbb{R}^{d}\right)$ such that $\lambda(\alpha V)\left\|u_{\alpha}\right\|_{d}^{d}=Q_{\alpha V}\left[u_{\alpha}\right]$.

Proof. Inequality (4.5) with $\beta$ large enough shows that $\lambda(\alpha V)<0$ for all $\alpha>0$. Hence the existence of a non-negative minimizer $u_{\alpha}$ follows from Lemma 4.1 in the same way as in the case $d<p$. Since $u_{\alpha}$ is a non-negative weak solution of (1.3), the Harnack inequality [S1, Thm. 6] implies that $u_{\alpha}$ is locally bounded and positive.

\subsection{Proof of Theorem 2.2, Lower bound.}

The case of positive $V$.

Proposition 4.4. Assume that $0 \leq V \in L^{q}\left(\mathbb{R}^{d}\right) \cap L^{1}\left(\mathbb{R}^{d}\right)$ for some $q>1$ with $V \not \equiv 0$. Then there are $\alpha_{0}>0$ and $C>0$ such that for all $0<\alpha \leq \alpha_{0}$ we have

$$
\lambda(\alpha V) \geq-C \alpha^{-1} \exp \left[-\left(\frac{d^{d-1} \omega_{d}}{\alpha \int_{\mathbb{R}^{d}} V d x}\right)^{\frac{1}{d-1}}\right] .
$$

Proof. Let $V^{*}$ be the symmetric decreasing rearrangement of $V$. Since $\int_{\mathbb{R}^{d}} V d x=\int_{\mathbb{R}^{d}} V^{*} d x$, $\int_{\mathbb{R}^{d}} V^{q} d x=\int_{\mathbb{R}^{d}}\left(V^{*}\right)^{q} d x$ and, by rearrangement inequalities (see, e.g., [Ta] and [LL, Thm. $3.4]$ ),

$$
\lambda(\alpha V) \geq \lambda\left(\alpha V^{*}\right),
$$

we may and will assume in the following that $V=V^{*}$.

By Corollary 4.3 there is a minimizer $u_{\alpha}$ of $Q_{\alpha V}[u] /\|u\|_{d}^{d}$. Again, by rearrangement inequalities, we may assume that $u_{\alpha}$ is a radially symmetric function which is non-increasing with respect to the radius. Let $\rho>0$ be an arbitrary parameter. (In this proof there is no 
loss in assuming that $\rho=1$, but in the proof of Proposition 4.5 we will repeat the argument with a general $\rho$.) We normalize $u_{\alpha}$ such that

$$
u_{\alpha}(x)=u_{\alpha}(|x|)=1, \quad \text { for all } x \in \mathbb{R}^{d} \text { with }|x|=\rho .
$$

Let $R \geq 2 \rho$ be a parameter to be specified later and let $\chi$ be defined by

$$
\chi(r)=1 \quad \text { if } \quad 0 \leq r \leq \rho, \quad \chi(r)=\left(1-\frac{r-\rho}{R-\rho}\right)_{+} \quad \text { if } r>\rho .
$$

Then for any $\varepsilon \in(0,1]$ we have

$$
\begin{aligned}
\left\|\nabla\left(\chi u_{\alpha}\right)\right\|_{d}^{d} & \leq(1+\varepsilon)\left\|\chi \nabla u_{\alpha}\right\|_{d}^{d}+c \varepsilon^{1-d}\left\|u_{\alpha} \nabla \chi\right\|_{d}^{d} \\
& \leq(1+\varepsilon)\left\|\nabla u_{\alpha}\right\|_{d}^{d}+c^{\prime} \varepsilon^{1-d} R^{-d}\left\|u_{\alpha}\right\|_{d}^{d},
\end{aligned}
$$

and therefore

$$
\left\|\nabla u_{\alpha}\right\|_{d}^{d} \geq\left\|\nabla\left(\chi u_{\alpha}\right)\right\|_{d}^{d} /(1+\varepsilon)-c^{\prime \prime} \varepsilon^{1-d} R^{-d}\left\|u_{\alpha}\right\|_{d}^{d} .
$$

Since $\chi u_{\alpha}$ has support in the ball of radius of radius $R$ and is bounded from below by one on the ball of radius $\rho$, the formula for the capacity of two nested balls [M, Sec. 2.2.4] gives

$$
\left\|\nabla u_{\alpha}\right\|_{d}^{d} \geq \frac{\omega_{d}(\log (R / \rho))^{1-d}}{1+\varepsilon}-c^{\prime \prime} \varepsilon^{1-d} R^{-d}\left\|u_{\alpha}\right\|_{d}^{d} .
$$

Moreover, since $\left|u_{\alpha}(x)\right| \leq 1$ for $|x|>1$, we obtain

$$
\lambda(\alpha V) \geq \frac{\omega_{d}(\log (R / \rho))^{1-d}-(1+\varepsilon) \alpha\left(\int_{B_{1}} V u_{\alpha}^{d} d x+\int_{B_{1}^{c}} V d x\right)}{(1+\varepsilon)\left\|u_{\alpha}\right\|_{d}^{d}}-\frac{c^{\prime \prime}}{\varepsilon^{d-1} R^{d}} .
$$

We next claim that there are constants $C>$ and $\alpha_{0}>0$ such that for all $0<\alpha \leq \alpha_{0}$,

$$
\sup _{B_{\rho}}\left(u_{\alpha}^{d}-1\right) \leq C \alpha^{\frac{1}{d-1}}
$$

Accepting this for the moment and returning to (4.9) we obtain

$$
\lambda(\alpha V) \geq \frac{\omega_{d}(\log (R / \rho))^{1-d}-(1+\varepsilon)\left(1+C \alpha^{\frac{1}{d-1}}\right) \alpha \int_{\mathbb{R}^{d}} V d x}{(1+\varepsilon)\left\|u_{\alpha}\right\|_{d}^{d}}-\frac{c^{\prime \prime}}{\varepsilon^{d-1} R^{d}} .
$$

For given $0<\varepsilon \leq 1$ and $0<\alpha \leq \alpha_{0}$ we now choose

$$
R=\rho \exp \left(\left(\frac{\omega_{d}}{(1+\varepsilon)\left(1+C \alpha^{\frac{1}{d-1}}\right) \alpha \int_{\mathbb{R}^{d}} V d x}\right)^{\frac{1}{d-1}}\right)
$$

so that

$$
\lambda(\alpha V) \geq-\frac{c^{\prime \prime}}{\varepsilon^{d-1} \rho^{d}} \exp \left(-d\left(\frac{\omega_{d}}{(1+\varepsilon)\left(1+C \alpha^{\frac{1}{d-1}}\right) \alpha \int_{\mathbb{R}^{d}} V d x}\right)^{\frac{1}{d-1}}\right)
$$

Finally, we choose $\varepsilon=C \alpha^{\frac{1}{d-1}}$ to obtain

$$
\lambda(\alpha V) \geq-\frac{c^{\prime \prime \prime}}{\alpha} \exp \left(-d\left(\frac{\omega_{d}}{\left(1+C^{\prime} \alpha^{\frac{1}{d-1}}\right) \alpha \int_{\mathbb{R}^{d}} V d x}\right)^{\frac{1}{d-1}}\right) .
$$

Up to increasing $c^{\prime \prime \prime}$ this implies the statement of the proposition. 
Thus, it remains to prove (4.10). For simplicity we give the proof only for $\rho=1$ (which is enough for the proof of the proposition). We apply Alvino's version of the Moser-Trudinger inequality Al] to the function $u_{\alpha}-1$ and obtain

$$
0<u_{\alpha}(r)-1 \leq C\left\|\nabla u_{\alpha}\right\|_{L^{d}\left(B_{1}\right)}|\log r|^{\frac{d-1}{d}}, \quad r \leq 1 .
$$

Using this upper bound on $u_{\alpha}$ we arrive at

$$
\begin{aligned}
\left\|\nabla u_{\alpha}\right\|_{L^{d}\left(B_{1}\right)}^{d} & \leq\left\|\nabla u_{\alpha}\right\|_{d}^{d} \\
& \leq \alpha \int_{\mathbb{R}^{d}} V\left|u_{\alpha}\right|^{d} d x \\
& \leq \alpha 2^{d-1}\left(\|V\|_{L^{1}\left(B_{1}\right)}+C\left\|\nabla u_{\alpha}\right\|_{L^{d}\left(B_{1}\right)}^{d} \omega_{d} \int_{0}^{1} V(r)|\log r|^{d-1} r^{d-1} d r\right) .
\end{aligned}
$$

The assumption $V \in L^{q}\left(\mathbb{R}^{d}\right)$ for some $q>1$ implies that $V \in L^{1}\left(B_{1},|\log | x||^{d-1} d x\right)$, and therefore there is a $C^{\prime}>0$ and an $\alpha_{0}>0$ such that for all $0<\alpha \leq \alpha_{0}$

$$
\left\|\nabla u_{\alpha}\right\|_{L^{d}\left(B_{1}\right)}^{d} \leq C^{\prime} \alpha^{1 / d} \text {. }
$$

Re-inserting this into (4.12), we find for all $0<\alpha \leq \alpha_{0}$

$$
0<u_{\alpha}(r)-1 \leq C^{\prime \prime} \alpha^{1 / d}|\log r|^{\frac{d-1}{d}}, \quad r \leq 1 .
$$

Hence the minimizer $u_{\alpha}$ satisfies for all $0<r \leq 1$,

$$
\begin{aligned}
\left(\left(-r u_{\alpha}^{\prime}(r)\right)^{d-1}\right)^{\prime} & =\alpha V(r) u_{\alpha}(r)^{d-1} r^{d-1}+\lambda(\alpha) u_{\alpha}(r)^{d-1} r^{d-1} \\
& \leq \alpha V(r) r^{d-1}\left(1+C^{\prime \prime} \alpha^{\frac{1}{d}}|\log r|^{\frac{d-1}{d}}\right)^{d-1}
\end{aligned}
$$

and

$$
\begin{aligned}
\left(\left(-r u_{\alpha}^{\prime}(r)\right)^{d-1}\right)^{\prime} & =\alpha V(r) u_{\alpha}(r)^{d-1} r^{d-1}+\lambda(\alpha) u_{\alpha}(r)^{d-1} r^{d-1} \\
& \geq \lambda(\alpha) r^{d-1}\left(1+C^{\prime \prime} \alpha^{\frac{1}{d}}|\log r|^{\frac{d-1}{d}}\right)^{d-1} .
\end{aligned}
$$

Since the right hand sides of (4.14) and (4.15) are integrable with respect to $r$ (for (4.14) we use here again the assumption that $V \in L^{1}\left(\mathbb{R}^{d}\right) \cap L^{q}\left(\mathbb{R}^{d}\right)$ for some $\left.q>1\right)$, the function $\left(-r u_{\alpha}^{\prime}(r)\right)^{d-1}$ has a finite limit as $r \rightarrow 0$. Since $u_{\alpha} \in W^{1, d}\left(\mathbb{R}^{d}\right)$, it follows that this limit must be zero. Thus, from (4.14) we get for all $0<r \leq 1$

$$
\begin{aligned}
\left(-r u_{\alpha}^{\prime}(r)\right)^{d-1} & \leq \alpha \int_{0}^{r} V(s) s^{d-1}\left(1+C^{\prime \prime} \alpha^{\frac{1}{d}}|\log s|^{\frac{d-1}{d}}\right)^{d-1} d s \\
& \leq \alpha\|V\|_{L^{q}\left(B_{1}\right)}\left(\int_{0}^{r} s^{d-1}\left(1+C^{\prime \prime} \alpha^{\frac{1}{d}}|\log s|^{\frac{d-1}{d}}\right)^{q^{\prime}(d-1)} d s\right)^{1 / q^{\prime}} \\
& \leq C^{\prime \prime \prime} \alpha\|V\|_{L^{q}\left(B_{1}\right)} r^{d / q^{\prime}}(1+|\log r|)^{\frac{(d-1)^{2}}{d}} .
\end{aligned}
$$

Finally, this implies that

$$
\begin{aligned}
u_{\alpha}(r)-1 & =-\int_{r}^{1} u_{\alpha}^{\prime}(s) d s \\
& \leq\left(C^{\prime \prime \prime} \alpha\|V\|_{L^{q}\left(B_{1}\right)}\right)^{\frac{1}{d-1}} \int_{r}^{1} s^{\frac{d}{q^{\prime}(d-1)}}(1+|\log s|)^{\frac{(d-1)}{d}} \frac{d s}{s} .
\end{aligned}
$$


Since the integral on the right side converges, we have shown (4.10). This completes the proof of the lemma.

The case of compactly supported $V$.

Proposition 4.5. Let $V$ be a function with compact support, $\int_{\mathbb{R}^{d}} V(x) d x>0$ and $V \in$ $L^{q}\left(\mathbb{R}^{d}\right)$ for some $q>1$. Then there are $\alpha_{0}>0$ and $C>0$ such that for all $0<\alpha \leq \alpha_{0}$ we have

$$
\lambda(\alpha V) \geq-\exp \left[-\left(\frac{d^{d-1} \omega_{d}}{\alpha \int_{\mathbb{R}^{d}} V d x\left(1+C \alpha^{\frac{1}{d}}\right)}\right)^{\frac{1}{d-1}}\right] .
$$

Similarly as in the case $d<p$ a key ingredient in the proof is to show that minimizers, when suitably normalised, converge locally to a constant function. In the case $d<p$ we deduced this from Morrey's inequality. Here the argument is considerably more complicated and based on Harnack's inequality for quasi-linear equations. We shall prove

Lemma 4.6. For each $d \in \mathbb{N}, q>1$ and $M>0$ there are constants $C>0$ and $\beta \in(0,1)$ with the following property. Let $\rho>0$ and assume that $W \in L_{\text {loc }}^{q}\left(\mathbb{R}^{d}\right)$ with $W \leq 0$ in $B_{5 \rho}^{c}$ and $\rho^{d-\frac{d}{q}}\|W\|_{L^{q}\left(B_{15 \rho}\right)} \leq M$. Then, if $u \in W^{1, d}\left(\mathbb{R}^{d}\right)$ is a positive, weak solution of the equation $-\Delta_{d}(u)=W u^{d-1}$ in $\mathbb{R}^{d}$ satisfying $\inf _{B_{5 \rho}} u \leq 1$ and if $y \in \mathbb{R}^{d}$ and $r>0$ are so that $B(3 r, y) \subset B_{3 \rho}$, we have

$$
\sup _{B(r, y)} u-\inf _{B(r, y)} u \leq C\|W\|_{L^{q}\left(B_{5 \rho}\right)}^{1 / d} \rho^{1-\frac{1}{q}-\beta} r^{\beta} .
$$

The point of this lemma is that the dependence of $W$ enters explicitly on the right side of (4.17). In our application, we will have $\|W\|_{L^{q}\left(B_{5 \rho}\right)} \rightarrow 0$, and therefore Lemma 4.6 shows that the oscillations of $u$ vanish with an explicit rate.

We recall that $u$ is a weak solution of $-\Delta_{d}(u)=W|u|^{d-2} u$ in $\mathbb{R}^{d}$ if

$$
\int_{\mathbb{R}^{d}}|\nabla u|^{d-2} \nabla u \cdot \nabla \varphi d x=\int_{\mathbb{R}^{d}} W|u|^{d-2} u \varphi d x
$$

for any $\varphi \in W^{1, d}\left(\mathbb{R}^{d}\right)$.

The following lemma, whose proof can be found, for instance, in Mo1, Mo2] or [LU, Lem. 2.4.1], plays a key role in the proof of Lemma 4.6.

Lemma 4.7. Let $\Omega \subseteq \mathbb{R}^{d}$ be open and assume that $u \in W^{1, d}(\Omega)$ is such that there are constants $K>0$ and $\beta>0$ such that for all $y \in \Omega$ and $r>0$ with $B(r, y) \subset \Omega$ one has

$$
\int_{B(r, y)}|\nabla u|^{d} d x \leq K r^{\beta d} .
$$

Then for all $y \in \Omega$ and $r>0$ such that $B(3 r / 2, y) \subset \Omega$ we have

$$
\sup _{B(r / 2, y)} u-\inf _{B(r / 2, y)} u \leq \frac{4}{\beta}\left(\frac{K}{\omega_{d}}\right)^{\frac{1}{d}} r^{\beta} .
$$

Proof of Lemma 4.6. By the Harnack inequality [S1, Thm.6] there is a constant $C_{1}$, which depends only on $d, q$ and an upper bound on $\rho^{d-\frac{d}{q}}\|W\|_{L^{q}\left(B_{15 \rho}\right)}$ such that

$$
\sup _{B_{5 \rho}} u \leq C_{1} \inf _{B_{5 \rho}} u \text {. }
$$


Since $\inf _{B_{5 \rho}} u(x) \leq 1$, we conclude that

$$
\sup _{B_{5 \rho}} u(x) \leq C_{1}
$$

Our goal is to apply Lemma 4.7 with $\Omega=B_{3 \rho}$. We have to verify condition (4.19) for some $K$ and $\beta$. First, note that

$$
\int_{\mathbb{R}^{d}}|\nabla u|^{d} d x=\int_{\mathbb{R}^{d}} W u^{d} d x \leq \int_{B_{5 \rho}} W u^{d} d x \leq \omega_{d}^{1-\frac{1}{q}}(5 \rho)^{d-\frac{d}{q}}\|W\|_{L^{q}\left(B_{5 \rho}\right)} C_{1}^{d}=c_{1} \mathcal{N},
$$

where we have set $c_{1}=\omega_{d}^{1-\frac{1}{q}} 5^{d-\frac{d}{q}}$ and

$$
\mathcal{N}=\rho^{d-\frac{d}{q}}\|W\|_{L^{q}\left(B_{5 \rho}\right)} C_{1}^{d} .
$$

Hence, for any $\beta>0$, (4.19) holds for any ball $B(r, y) \subset B_{3 \rho}$ with $r \geq \rho$ provided we choose the constan $K$ at least as big as $c_{1} \mathcal{N} \rho^{-\beta d}$.

Thus, it remains to verify (4.19) for $r<\rho$. Let $0 \leq \zeta \leq 1$ be a radial function with support in $\overline{B_{2}}$ which is $\equiv 1$ on $B_{1}$ and satisfies $|\nabla \zeta| \leq 1$. Let $y$ and $s$ be such that $B(2 s, y) \subset B_{5 \rho}$. We choose the test function $\varphi(x)=\zeta(|x-y| / s)(u(x)-a)$ in (4.18), where the parameter $a$ will be specified later. This gives the inequality

$$
\begin{aligned}
\int_{B(s, y)}|\nabla u|^{d} d x & \leq \int_{\mathbb{R}^{d}} \zeta(|x-y| / s)|\nabla u|^{d} d x \\
& \leq \int_{B(2 s, y)}|W| u^{d-1}|u-a| d x+s^{-1} \int_{A(s, y)}|\nabla u|^{d-1}|u-a| d x .
\end{aligned}
$$

with $A(s, y)=B(2 s, y) \backslash B(s, y)$. Now we set $a=\frac{1}{|A(s, y)|} \int_{A(s, y)} u d x$, where $|A(s, y)|$ denotes the Lebesgue measure of $A(s, y)$. By the Hölder and Poincaré inequalities,

$$
\begin{aligned}
\int_{A(s, y)}|\nabla u|^{d-1}|u-a| d x & \leq\left(\int_{A(s, y)}|\nabla u|^{d} d x\right)^{\frac{d-1}{d}}\left(\int_{A(s, y)}|u-a|^{d} d x\right)^{\frac{1}{d}} \\
& \leq C^{\mathrm{P}} s \int_{A(s, y)}|\nabla u|^{d} d x,
\end{aligned}
$$

where $C^{\mathrm{P}}$ is the constant in the Poincaré inequality in $A(1,0)$. By scaling one easily sees that the Poincaré constant in $A(s, y)$ is given by $C^{\mathrm{P}} s$. This fact was used in the previous bound.

Let us bound the first term on the right side of (4.24). Since both $u$ and $|a|$ are bounded from above by $C_{1}$ on $B(2 s, y)$, see (4.21), we have

$$
\int_{B(2 s, y)}|W| u^{d-1}|u-a| d x \leq\|W\|_{L^{1}(B(2 s, y))} 2 C_{1}^{p} \leq c_{2} \mathcal{N}(s / \rho)^{d-\frac{d}{q}},
$$

where $c_{2}=\omega_{d}^{1-\frac{1}{q}} 2^{d+1-\frac{d}{q}}$.

Thus, 4.24) implies

$$
\int_{B(s, y)}|\nabla u|^{d} d x \leq c_{2} \mathcal{N}(s / \rho)^{d-\frac{d}{q}}+C^{\mathrm{P}} \int_{A(s, y)}|\nabla u|^{d} d x
$$


where $c_{1}=2^{d+1-\frac{d}{q}} \omega_{d}^{1-\frac{1}{q}}$. Adding $C^{\mathrm{P}} \int_{B(s, y)}|\nabla u|^{d} d x$ to both sides of the above inequality we arrive at

$$
\int_{B(s, y)}|\nabla u|^{d} d x \leq c_{3} \mathcal{N}(s / \rho)^{d-\frac{d}{q}}+\kappa \int_{B(2 s, y)}|\nabla u|^{d} d x
$$

with $c_{3}=c_{2} /\left(1+C^{\mathrm{P}}\right)$ and

$$
\kappa=\frac{C^{\mathrm{P}}}{1+C^{\mathrm{P}}}<1
$$

To simplify the notation, we introduce the shorthand $D(s)=\int_{B(s, y)}|\nabla u|^{d} d x$. Iterating inequality (4.25) gives

$$
D\left(2^{-n} s\right) \leq c_{3} \mathcal{N}(s / \rho)^{d-\frac{d}{q}} 2^{n\left(\frac{d}{q}-d\right)} \sum_{j=0}^{n-1}\left(\kappa 2^{d-\frac{d}{q}}\right)^{j}+\kappa^{n} D(s)
$$

for all $n \in \mathbb{N}$ and every $s>0$ such that $B(s, y) \subset B_{5 \rho}$. Next, we sum the geometric series on the right side and obtain a $c_{4}$ and a $\mu<1$ (both depending only on $d$ and $q$ ) such that

$$
2^{n\left(\frac{d}{q}-d\right)} \sum_{j=0}^{n-1}\left(\kappa 2^{d-\frac{d}{q}}\right)^{j} \leq c_{4} \mu^{n} \quad \text { for all } n \in \mathbb{N} .
$$

Thus, recalling (4.22),

$$
D\left(2^{-n} s\right) \leq\left(c_{3} c_{4}(s / \rho)^{d-\frac{d}{q}}+c_{1}\right) \mathcal{N} \max \left\{\mu^{n}, \kappa^{n}\right\}
$$

for all $n \in \mathbb{N}$ and all $s$ such that $B(s, y) \subset B_{5 \rho}$.

Now let $B(r, y) \subset B_{3 \rho}$ with $r<\rho$. There are $k \in \mathbb{N}$ and $t \in[1,2)$ such that $2^{-k-1} t \rho<$ $r \leq 2^{-k} t \rho$. Since $B(t \rho, y) \subset B_{5 \rho}$ we may apply inequality (4.26) with $k=n$ and $s=t \rho$ to get

$$
\begin{aligned}
\int_{B(r, y)}|\nabla u|^{d} d x & \leq D\left(2^{-k} t \rho\right) \\
& \leq\left(c_{3} c_{4} t^{d-\frac{d}{q}}+c_{1}\right) \mathcal{N} \max \left\{\mu^{k}, \kappa^{k}\right\} \\
& \leq\left(c_{3} c_{4} 2^{d-\frac{d}{q}}+c_{1}\right) \mathcal{N}\left(\frac{2 r}{\rho}\right)^{\beta d} \quad \text { with } \beta=-\frac{\log \max \{\mu, \kappa\}}{d \log 2}>0 .
\end{aligned}
$$

To summarize, we have shown that (4.19) holds for any $B(r, y) \subset B_{3 \rho}$ with the above choice of $\beta$ and with

$$
K=\max \left\{c_{1},\left(c_{3} c_{4} 2^{d-\frac{d}{q}}+c_{1}\right) 2^{\beta d}\right\} \mathcal{N} \rho^{-\beta d} .
$$

Here $c_{1}, c_{3}$ and $c_{4}$ depend only on $d$ and $q$, and $\mathcal{N}$ was defined in (4.23). In view of Lemma 4.7 this proves (4.17).

Proof of Proposition 4.5. The beginning of the proof is identical to that of Proposition 4.4, Let $\rho>0$ be such that the support of $V$ is contained in $\overline{B_{5 \rho}}$. We let again $u_{\alpha}$ be a minimizer of $Q_{\alpha V}[u] /\|u\|_{d}^{d}$. From Corollary 4.3 we know that $u_{\alpha}$ can be chosen strictly positive and therefore we may normalize $u_{\alpha}$ by $\inf _{B_{\rho}} u_{\alpha}=1$. Arguing exactly as before we arrive at the following variant of (4.9),

$$
\lambda(\alpha V) \geq \frac{\omega_{d}(\log (R / \rho))^{1-d}-(1+\varepsilon) \alpha \int_{\mathbb{R}^{d}} V\left|u_{\alpha}\right|^{d} d x}{(1+\varepsilon)\left\|u_{\alpha}\right\|_{d}^{d}}-c^{\prime \prime} \varepsilon^{1-d} R^{-d} .
$$


We now claim that there is a constant $C>0$ (depending on $d, q, V$, but not on $\alpha$ ) such that

$$
\left|u_{\alpha}(x)-1\right| \leq C \alpha^{\frac{1}{d}} \quad \text { for all } x \in B_{\rho} .
$$

Indeed, this follows from Lemma4.6 applied to $W=\alpha V+\lambda(\alpha V)$ and $u=u_{\alpha}$ with $B(r, y)=$ $B_{\rho}$. Note that we indeed have $\inf _{B_{5 \rho}} u_{\alpha} \leq \inf _{B_{\rho}} u_{\alpha}=1$. Moreover, we use the fact that $\lambda(\alpha V) \geq-C \alpha$, which follows easily from the bounds in Lemma 4.1.

With a similar choice as in Lemma 4.4 for $R$ we obtain

$$
\lambda(\alpha V) \geq-\frac{c^{\prime \prime}}{\varepsilon^{d-1} \rho^{d}} \exp \left(-d\left(\frac{\omega_{d}}{(1+\varepsilon)\left(1+C \alpha^{\frac{1}{d}}\right) \alpha \int_{\mathbb{R}^{d}} V d x}\right)^{\frac{1}{d-1}}\right)
$$

Choosing $\varepsilon=C \alpha^{\frac{1}{d}}$ we obtain

$$
\lambda(\alpha V) \geq-\frac{c^{\prime \prime \prime}}{\alpha^{\frac{d-1}{d}}} \exp \left(-d\left(\frac{\omega_{d}}{\left(1+C^{\prime} \alpha^{\frac{1}{d}}\right) \alpha \int_{\mathbb{R}^{d}} V d x}\right)^{\frac{1}{d-1}}\right) .
$$

This implies the statement of the proposition.

The general case. We can finally give the

Proof of Theorem 2.2. We use an approximation argument and fix $\varepsilon \in(0,1)$ and $R>0$. Define $V_{<}=V \chi_{\{|\cdot|<R\}}$ and $V_{>}=V_{+} \chi_{\{|\cdot| \geq R\}}$. Then the inequality

$$
Q_{\alpha V}[u] \geq(1-\varepsilon) Q_{(1-\varepsilon)^{-1} \alpha V_{<}}[u]+\varepsilon Q_{\varepsilon^{-1} \alpha V_{>}}[u]
$$

for every $u \in W^{1, d}\left(\mathbb{R}^{d}\right)$ implies

$$
\lambda(\alpha V) \geq(1-\varepsilon) \lambda\left(\frac{\alpha}{1-\varepsilon} V_{<}\right)+\varepsilon \lambda\left(\frac{\alpha}{\varepsilon} V_{>}\right) .
$$

Thus,

$$
\begin{aligned}
\log \frac{1}{|\lambda(\alpha V)|} & \geq \log \frac{1}{(1-\varepsilon)\left|\lambda\left((1-\varepsilon)^{-1} \alpha V_{<}\right)\right|}-\log \left(1+\frac{\varepsilon\left|\lambda\left(\varepsilon^{-1} \alpha V_{>}\right)\right|}{(1-\varepsilon)\left|\lambda\left((1-\varepsilon)^{-1} \alpha V_{<}\right)\right|}\right) \\
& \geq \log \frac{\varepsilon\left|\lambda\left(\varepsilon^{-1} \alpha V_{>}\right)\right|}{(1-\varepsilon)\left|\lambda\left((1-\varepsilon)^{-1} \alpha V_{<}\right)\right|}-\frac{1}{(1-\varepsilon)\left|\lambda\left((1-\varepsilon)^{-1} \alpha V_{<}\right)\right|} .
\end{aligned}
$$

From now on we consider $R$ so large that $\int_{B_{R}} V d x>0$. It then follows from Proposition 4.5 that

$$
\liminf _{\alpha \rightarrow 0+} \alpha^{\frac{1}{d-1}} \log \frac{1}{(1-\varepsilon)\left|\lambda\left((1-\varepsilon)^{-1} \alpha V_{<}\right)\right|} \geq(1-\varepsilon)^{\frac{1}{d-1}} d \omega_{d}^{\frac{1}{d-1}}\left(\int_{B_{R}} V(x) d x\right)^{-\frac{1}{d-1}} .
$$

On the other hand, we recall from Proposition 4.6 that there are constants $C>0$ and $\alpha_{0}>0$ such that for all $0<\alpha \leq \alpha_{0} \varepsilon$,

$$
\lambda\left(\varepsilon^{-1} \alpha V_{>}\right) \geq-C \varepsilon \alpha^{-1} \exp \left(-\left(\frac{\varepsilon d^{d-1} \omega_{d}}{\alpha \int_{B_{R}^{c}} V_{+} d x}\right)^{\frac{1}{d-1}}\right)
$$


Moreover, we recall from Proposition 4.2 that for every $\delta \in(0,1)$ there are constants $C_{\delta}>0$ and $\alpha_{\delta}$ such that for all $0<\alpha \leq \alpha_{\delta}(1-\varepsilon)$,

$$
\lambda\left((1-\varepsilon)^{-1} \alpha V_{<}\right) \leq-(1-\varepsilon)^{-1} \alpha C_{\delta} \exp \left(-\left(\frac{(1-\varepsilon) d^{d-1} \omega_{d}}{\alpha(1-\delta) \int_{B_{R}} V d x}\right)^{\frac{1}{d-1}}\right) .
$$

Thus, for $\alpha \leq \min \left\{\alpha_{0} \varepsilon, \alpha_{\delta}(1-\varepsilon)\right\}$,

$$
\frac{\left|\lambda\left(\varepsilon^{-1} \alpha V_{>}\right)\right|}{\left|\lambda\left((1-\varepsilon)^{-1} \alpha V_{<}\right)\right|} \leq \frac{C \varepsilon(1-\varepsilon)}{C_{\delta} \alpha^{2}} \exp \left(-\left(\frac{\varepsilon d^{d-1} \omega_{d}}{\alpha \int_{B_{R}^{c}} V_{+} d x}\right)^{\frac{1}{d-1}}+\left(\frac{(1-\varepsilon) d^{d-1} \omega_{d}}{\alpha(1-\delta) \int_{B_{R}} V d x}\right)^{\frac{1}{d-1}}\right)
$$

For every fixed $\varepsilon$ and $\delta$ there is an $R_{0}>0$ such that for all $R>R_{0}$,

$$
\frac{\varepsilon}{\int_{B_{R}^{c}} V_{+} d x}>\frac{1-\varepsilon}{(1-\delta) \int_{B_{R}} V d x} \text {. }
$$

Thus, for all $R>R_{0}$ we have

$$
\lim _{\alpha \rightarrow 0} \frac{\left|\lambda\left(\varepsilon^{-1} \alpha V_{>}\right)\right|}{\left|\lambda\left((1-\varepsilon)^{-1} \alpha V_{<}\right)\right|}=0 .
$$

To summarize, we have shown that for all $\varepsilon \in(0,1)$ and for all $R>R_{0}$,

$$
\liminf _{\alpha \rightarrow 0+} \alpha^{\frac{1}{d-1}} \log \frac{1}{|\lambda(\alpha V)|} \geq(1-\varepsilon)^{\frac{1}{d-1}} d \omega_{d}^{\frac{1}{d-1}}\left(\int_{B_{R}} V(x) d x\right)^{-\frac{1}{d-1}} .
$$

Letting $\varepsilon \rightarrow 0$ and $R \rightarrow \infty$ we obtain the theorem.

\section{ACKNOWLEDGEMENTS}

Partial financial support through Swedish research council grant FS-2009-493 (T. E.), U.S. National Science Foundation grant PHY-1347399 (R. F.) and grant MIUR-PRIN08 grant for the project "Trasporto ottimo di massa, disuguaglianze geometriche e funzionali e applicazioni" (H. K.) is acknowlegded.

\section{REFERENCES}

[Ad] R. A. Adams, J. J. F. Fournier, Sobolev spaces. Second edition. Pure and Applied Mathematics (Amsterdam), 140. Elsevier/Academic Press, Amsterdam, 2003. xiv+305 pp. ISBN: 0-12-044143-8

[Al] A. Alvino: Un caso limite della diseguaglianza di Sobolev in spazi di Lorentz, Rend. Acc. Sci. Fis. Mat. Napoli

[AZ1] J. Arazy, L. Zelenko: Virtual eigenvalues of the high order Schrödinger operator. I, Integral Equations Op. Theory 50 (2006) 189-231.

[AZ2] J. Arazy, L. Zelenko: Virtual eigenvalues of the high order Schrödinger operator. II, Integral Equations Op. Theory 50 (2006) 305-345.

[BCEZ] F. Bentosela, R. M. Cavalcanti, P. Exner, V. A. Zagrebnov, Anomalous electron trapping by localized magnetic fields. J. Phys. A 32 (1999), no. 16, 3029-3039.

[BGS] R. Blankenbecler, M. L. Goldberger, and B. Simon: The bound states of weakly coupled long-range one-dimensional quantum Hamiltonians, Ann. of Physics 108 (1977) 69-78.

[FMV] R.L. Frank, S. Morozov and S. Vugalter: Weakly coupled bound states of Pauli operators, Calc. Var. Partial Diff. Eq. 40 (2011) 253-271.

[GS] J. Garcia-Melian and J. Sabina de Lis: Maximum and comparison principles for operators involving the p-Laplacian, J. Math. Anal. Appl. 218 (1998) 49-65. 
[Ha] A.N. Hatzinikitas: The weakly coupled fractional one-dimensional Schrödinger operator with index $1<\alpha \leq 2$, J. Math. Phys. 51 (2010) 123523.

[Kl1] M. Klaus: On the bound state of Schrödinger operators in one dimension, Ann. of Physics 108 (1977) 288-300.

[Kl2] M. Klaus: A remark about weakly coupled one-dimensional Schrödinger operators, Helv. Phys. Acta 52 (1979) 223-229.

[KS] M. Klaus, B. Simon: Coupling constant thresholds in nonrelativistic quantum mechanics. I. Shortrange two-body case, Ann. of Physics 130 (1980) 251-281.

[LU] O. Ladyzhenskaya, N. Uraltseva: Linear and Quasilinear Elliptic Equations, Academic Press, New York 1968.

[LL] E. H. Lieb, M. Loss, Analysis. Second edition. Graduate Studies in Mathematics 14, American Mathematical Society, Providence, RI, 2001.

[M] W. Mazya: Sobolev spaces. Translated from the Russian by T. O. Shaposhnikova. Springer Series in Soviet Mathematics. Springer-Verlag, Berlin, 1985.

[Mo1] C. B. Morrey: On the solutions of quasilinear elliptic partial differential equations, Trans. Amer. Math. Soc. 43 (1938) 126-166.

[Mo2] C. B. Morrey: Multiple integral problems in the calculus of variations and related topics, Univ. of California Publ. 1 (1943) 1-130.

[OK] B. Opic, A. Kufner: Hardy-type inequalities. Pitman Research Notes in Mathematics Series, 219. Longman Scientific \& Technical, Harlow, 1990.

[PT1] Y. Pinchover, K. Tintarev: Ground state alternative for p-Laplacian with potential term, Calc. Var. Partial Diff. Eq. 25 (2007) 179-201.

[PT2] Y. Pinchover, K. Tintarev: On positive solutions of minimal growth for singular p-Laplacian with potential term, Advanced Nonlinear Studies 8 (2008), 213-234.

[PTT] Y. Pinchover, A. Tertikas and K. Tintarev: A Liouville-type theorem for the p-Laplacian with potential term, Ann. Inst. H. Poincare-Anal. Non Lineaire 25 (2008), 357-368.

[PoSh] A. Poliakovsky, I. Shafrir: Uniqueness of positive solutions for singular problems involving the pLaplacian, Proc. Amer. Math. Soc. 133 (2005), 2549-2557.

[PS] P. Pucci, J. Serrin: The Maximum Principle, Progress in Nonlinear Differential Equations and their Applications 73, Birkhäuser-Verlag, Basel, 2007.

[S1] J. Serrin: Local behavior of solutions of quasi-linear equations, Acta Math. 111 (1964) $247-302$.

[S2] J. Serrin: Isolated singularities of solutions of quasilinear equation, Acta Math. 113 (1965) $219-240$.

[Si] B. Simon: The Bound State of Weakly Coupled Schrödinger Operators in One and Two Dimensions, Ann. of Physics 97 (1976) 279-288.

[Ta] G. Talenti: Best constants in Sobolev inequality, Ann. Mat. Pura Appl. 110 (1976) 353-372.

[TT] P. Takáč, K. Tintarev: Generalized minimizer solutions for equations with the p-Laplacian and a potential term, Proc. Roy. Soc. Edinburgh Sect. A 138 (2008), 201-221.

[To] P. Tolksdorf: Regularity for a more general class of quasilinear elliptic equations, J. Differential Equations 51 (1984) 126-150.

[Tr] N.S. Trudinger: On Harnack Type Inequalities and Their Application to Quasilinear Elliptic Equations, Comm. Pure Appl. Math. 20 (1967) 721-747.

Tomas Ekholm, Department of Mathematics, Royal Institute of Technology, S-100 44 StockHOLM, SWEDEN

E-mail address: tomase@math.kth.se

Rupert L. Frank, Mathematics 253-37, Caltech, Pasadena, CA 91125, USA

E-mail address: rlfrank@caltech.edu

Hynek Kovařík, DiCATAM, Sezione di Matematica, Università degli studi di Brescia, Via Branze, 38 - 25123 Brescia, Italy

E-mail address: hynek.kovarik@ing.unibs.it 